\title{
High-resolution magnetic mapping of PR-interval phenomena of normal subjects
}

\author{
B. J. ten Voorde M.J. Peters \\ Low Temperature Physics Department, Twente University of Technology, PO Box 217. \\ 7500 AE Enschede. The Netherlands \\ G. Stroink \\ Department of Physics, Dalhousie University, Halifax, Nova Scotia B3H 4H2, Canada
}

L. R. van der Wieken

Hospital Ziekenzorg. Enschede. The Netherlands

\begin{abstract}
In this experimental study we measured the magnetocardiograms (MCGs) of 17 normals at 42 measuring sites in a plane near the chest. The recordings were made in an unshielded environment with a single channel second-order SOUID gradiometer. From these recordings spatial maps were constructed of the atrial depo larisation and repolarisation. The two series of maps were found to be dipolar, but opposite in sign, for 14 of the 17 subjects. The relatively small change in the ampli: tude and pattern of the atrial repolarisation maps built up our confidence in the method of subtracting a computed mean repolarisation map from the maps obtained during the last $40 \mathrm{~ms}$ before the ORS-onset. This method of separating His-Purkinje activity from atrial repolarisation led to distinct dipolar patterns in 9 out of the 17 cases. The direction and location of the associated electric current dipole was in good agreement with what can be expected electrophysiologically for His-Purkinje activation.
\end{abstract}

Keywords-Atrial repolarisation, High-resolution magnetocardiography. HisPurkinje, Magnetic isofield contour maps, PR-interval

Med. \& Biol Eng. \& Comput, 1988, 26, 130-135

\section{Introduction}

THE STUDY of specialised and relatively well localised conduction tissues of the heart, such as the His-Purkinje System (HPS), has been one of the main objectives of highresolution magnetocardiography over the last six years. The first magnetic recording of HPS-activity was reported by FARRell et al. in 1978. About a decade earlier Scherlag succeeded in demonstrating HPS activity in man by using an intracardiac catheter technique (SCHERLAG et al., 1969). In their electrical recordings a spike appeared about $40 \mathrm{~ms}$ prior to the onset of the QRS-complex. Four years later Berbari reported that HPS activity could be recorded by surface electrodes using high amplification, proper filtering and averaging techniques (BERBARI et al., 1973). The same group also demonstrated that by using a bandwidth of $0 \cdot 1-300 \mathrm{~Hz}$ the PR-segment showed 'ramp' patterns which commenced about $40 \mathrm{~ms}$ before the onset of QRS and represented HPS-activation.

In 1980 Farrell and his group also demonstrated the ramp pattern by means of high-resolution MCG (FARRELL et al., 1980). In the same year Fenici published results that

Dr ten Voorde is now at the Laboratory of Medical Physics, Free University, PO Box 7161, 1007 MC Amsterdam, The Netherlands.

First received 30th December 1986 and in final form 29th October 1987

(C) IFMBE: 1988 showed a similar ramp structure with certain 'bumps' in the PR-segment (FENICI et al., 1980). Both groups, measuring in an unshielded environment, considered the ramps to be due to continuous activation of the HPS. In later work done in the Berlin Magnetically Shielded Room, the highresolution magnetic data did not always show a clear division of two regions with positive and negative ramps, as reported in the papers mentioned above. In only 10 out of 32 cases single definite 'bumps' superimposed on the ramps, which could be related to the His-bundle activation, were found (ERNÉ et al., 1984b). It could be argued that looking only at single high-resolution MCG or ECG time recordings and interpreting certain 'bumps' or 'ramps' as representing HPS activity is a somewhat doubtful technique. It is difficult to decide which bump or which ramp would be HPS-activity because the PR-intervals show many wavelets and there are no objective criteria to pick out the 'right' ones.

A better method of interpreting the HR-MCG data might be to combine all 42 PR-segment tracings into a sequence of spatial maps. Only when the magnetic field distribution shows meaningful dipolar patterns in the sense that associated current dipoles have a location and direction which can be expected from physiological knowledge and simulations, can one interpret this magnetic field as being caused by HPS depolarisation. Simulation studies of the HPS activation do show dipolar patterns in the magnetic field distribution (ERNÉ et al., 1985a). 
There remains one difficulty to overcome. Some method has to be found to reliably separate the signal due to the atrial repolarisation from that produced by the HPS activation, as both signals overlap each other in time. Several atrial repolarisation 'subtracting' techniques have been suggested. These are

(a) subtracting the magnetic field pattern found just after the $\mathbf{P}$-wave offset from the signals obtained during the last part of the PR-segment (ERNÉ et al., 1984b; FENICI et al., 1985)

(b) removing the atrial repolarisation by cross-correlating the static repolarisation pattern with maps of the HPS activation as has been done in high-resolution ECG (HORAN et al., 1982)

(c) separating repolarisation from HPS activity by an expansion technique using MCG-spatial eigenfunctions representing the atrial repolarisation pattern (MACAulay et al., 1985).

Lately it has been proved that the last and the first method give similar results (LAMOTHE et al., 1987), which favours our more straightforward method of subtracting a mean atrial repolarisation pattern.

The aim of our research was to address in a nonshielded environment the following question: Is it possible by means of mapping the magnetic field over the entire chest to distinguish between atrial depolarisation, atrial repolarisation, HPS activity and very early septal depolarisation?

\section{Methods}

\subsection{SQUID-magnetometer}

The high-resolution MCGs were recorded with a singlechannel magnetometer consisting of a BTi RF-SQUID coupled to a homemade second-order gradiometer. The gradiometer had a baseline of $4 \mathrm{~cm}$ and a diameter of $3 \mathrm{~cm}$ and was balanced with a three-dimensional system of adjustable superconducting vanes. SQUID and gradiometer were kept superconductive in a 51 fibreglass dewar containing liquid helium. The recordings were performed in an unshielded room in a small brick building on the university campus during daytime. Before digitising the MCG-signal it was filtered by an active notch filter to partially remove the $50 \mathrm{~Hz}$ line interference and a $24 \mathrm{~dB}$ octave $^{-1}$ low-pass filter with cutoff frequency of $100 \mathrm{~Hz}$.

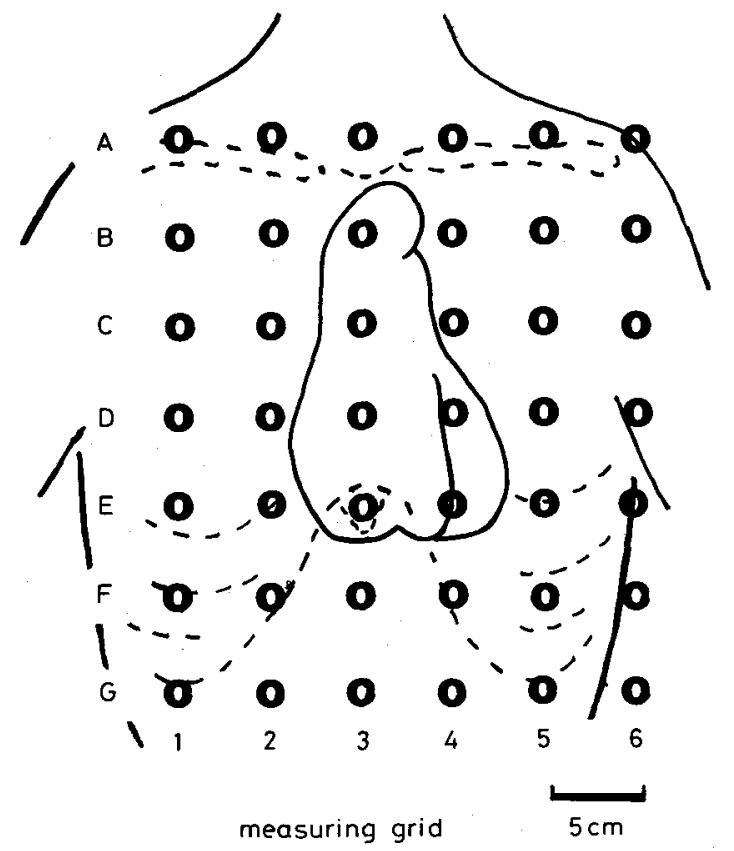

Fig. 1 Magnetocardiographic measuring grid
After this procedure the noise level was approximately 20 $35 \mathrm{fT} \mathrm{Hz}^{-1 / 2} \quad\left(\mathrm{fT}=\right.$ femtotesla $=10^{-15}$ tesla $)$. To avoid signal distortion no high-pass filters were used.

The magnetic field component normal to a plane that is tangential to the subject's chest has been recorded sequentially at 42 points forming a rectangular measuring grid. The 42 sites with grid space of $5 \mathrm{~cm}$ in both directions resulted in a total grid dimension of $25 \times 30 \mathrm{~cm}$ (Fig. 1). Rows are labelled $A-G$ and columns $1-6$. The subject was in the supine position during recording, with the measuring plane (i.e. the lowest coil of the gradiometer) as close to the subject's chest as possible, typically $15-20 \mathrm{~mm}$ from the chest. Fields coming out of the chest were defined as negative.

\subsection{Data acquisition and processing}

A 12-bit A/D convertor was used to digitise the data at a rate of $1000 \mathrm{~Hz}$. A finite impulse-response digital filter, with a cutoff frequency of $100 \mathrm{~Hz}$ and a notch at $50 \mathrm{~Hz}$, was applied to remove the remaining line interference frequencies. A PDP-11 73+ microcomputer system performed real-time averaging of heart beats centred in a $700 \mathrm{~ms}$ long window. As a trigger for aligning the MCG beats an analogue so-called 'dual-level-cross' QRS-detector was used. At a preset time delay after a QRS-peak has occurred the QRS detector sends a TTL pulse to the PDP-11. Peak detection is based on a level criterium for both ascending and descending slopes of the QRS-complex in the standard ECG-II limb lead, which was bandpass filtered between $0 \cdot 1$ and $30 \mathrm{~Hz}$. This method of aligning the MCG complexes is not affected by drift in the ECG reference lead. The trigger jitter of this hardware QRS-detector was about $0.2 \mathrm{~ms}$ (Ros, 1976). The real-time averaging process of the MCG beats was made visible on a video display and served as a useful tool to control continuously the noise level present in the MCG signal and to reject too noisy beats. Only 25 cardiac cycles were averaged at each grid point, which resulted in a very reasonable total recording time of about 60 minutes for each subject, including preparation for proper triggering on the ECG limb lead. When thought necessary the averaged data could be filtered offline with software filters.

To produce smooth isocontour maps at a certain time instant, the magnetic field was computed in a fine regular grid of $20 \times 20$ points from the 42 measured data, using a fifth-degree bivariate interpolation function (AKIMA, 1978). From this fine grid of 400 interpolated function values isocontour lines were drawn by linear interpolation (SNIJDER, 1978). Maps could be made every millisecond from $250 \mathrm{~ms}$ before QRS-onset to $450 \mathrm{~ms}$ after. All time instants mentioned in this article are related to the onset of the QRS-complex. The QRS onset was taken as the earliest ventricular depolarisation visible in any of the 42 high-resolution magnetic recordings.

Numerical calculations, based upon a volume conductor model which consisted of a realistically shaped torso with four compartments of different conductivity, representing the two lungs and the two intercavitary blood masses, showed that the general features of an isofield contour map were not much influenced by the volume conductor (PETERs et al., 1982). The contour indicating zero measured field almost coincided with the one calculated for a current dipole situated in an infinite homogeneous conductor. Although recent model studies report a change of the apparent dipolar source in the presence of intraventricular blood masses, the contributions are small enough to qualitatively interpret magnetocardiographic maps with the simplified infinite-volume-conductor model (HORACEK et al., 1986). Therefore by displaying in isofield contour maps 
the component of the magnetic field measured perpendicular to a plane parallel to the chest, we can make an estimation of depth, position and direction of the current dipole source which is parallel to this plane. To separate atrial repolarisation from HPS activation we computed a mean repolarisation map over a time segment just after P-wave offset. This offset was determined by means of visual inspection of the 42 magnetic recordings of each subject. The time segment taken was 10-25 ms long, depending on the length of the PR-segment of the subject.

\subsection{Subjects}

We measured 16 normal subjects with no history of heart disease and one male of 42 years old in whom the HPS conduction time (HV-time) was known by catheterisation. Five out of these 17 subjects were female. Ages ranged from 23 to 65 with an average of 35 years.

\section{Results}

Before discussing any details of the recordings the average amplitudes of the magnetic field component perpendicular to the chest of the main heart cycle complexes found for the 17 subjects should be noted. The standard deviation in picotesla (pT) is given in brackets. The positive deflections of the P-wave had a mean value of $2.5 \mathrm{pT}$ (1) and a maximum of $5 \mathrm{pT}$, whereas the negative $\mathrm{P}$ deflections had a mean value of $-4 \mathrm{pT}(1.5)$ and a maximum of $-7 \mathrm{pT}$. We found the mean $\mathrm{R}$-wave to be $52 \mathrm{pT}$ (17) with a maximum of $82 \mathrm{pT}$. The mean Samplitude was $22 \mathrm{pT}$ (7) and maximum $70 \mathrm{pT}$. The T-wave had an average positive deflection of 21 (7) and maximum of $35 \mathrm{pT}$ and a mean negative deflection of $13 \mathrm{pT}(5)$ and maximum of $25 \mathrm{pT}$. The RMS value of the noise measured in the baseline segments just before P-wave onset varied between 25 and $60 \mathrm{fT}$.
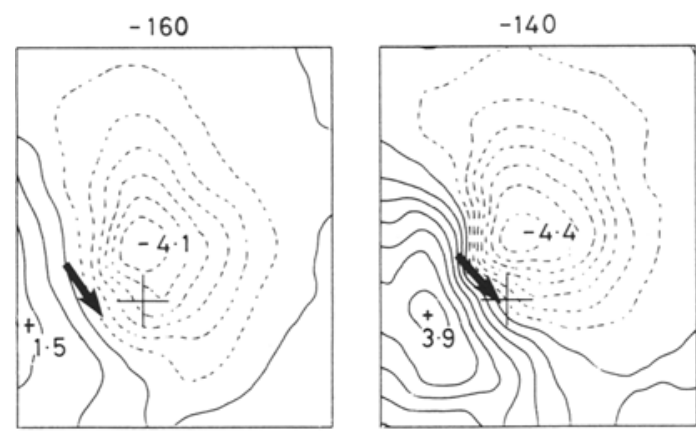

atrial depolarisation
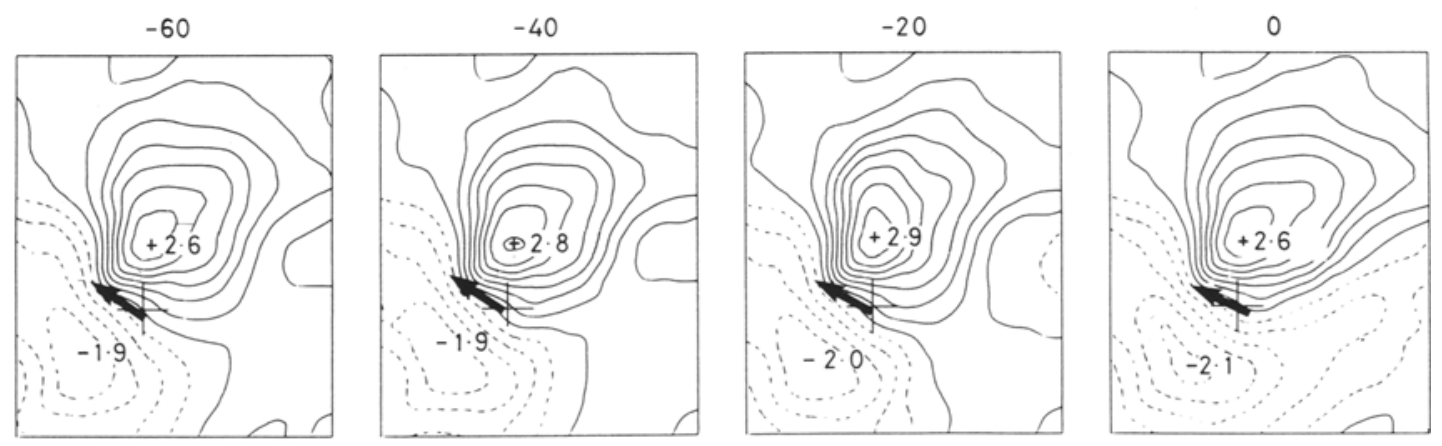

\subsection{Atrial depolarisation and repolarisation maps}

It was found that the spatial maps of atrial depolarisation of most of the subjects (11 of 17) have a clear dipolar pattern. The direction of the apparent current dipole is pointing down-leftwards along the heart axis and the projection of the location was always found between grid points D2-D3-E2-E3. These grid points are roughly above the atria. The field pattern of the other six subjects showed mainly negative values. Maps of atrial repolarisation were found to be dipolar in 14 out of 17 subjects, with the opposite polarity to the depolarisation maps. So the direction of the atrial repolarisation current dipole changes $180^{\circ}$ relative to the depolarisation activity. Localisation of the repolarisation current dipole appeared in the same grid area in which the depolarisation current dipole was found. Of the six subjects who showed mainly negative depolarisation patterns three also had a clear dipolar field during repolarisation. The remaining three had mainly positive repolarisation patterns. time variation of the magnetic field due to atrial activity. The top row maps are taken during atrial depolarisation, each $20 \mathrm{~ms}$ apart in time. The second row gives four maps during atrial repolarisation. Again the time interval between successive maps is $20 \mathrm{~ms}$. For the computation of all these maps the baseline is taken as the mean value of a $50 \mathrm{~ms}$ time segment prior to atrial activation.

\subsection{Atrial repolarisation and HPS activation maps} tion during the PR-segment, only relatively small changes in both amplitudes and patterns were found in all subjects (Figs. 2 and 3). The amplitude variation of the repolarisation patterns in all subjects was on average less than $200 \mathrm{fT}$, representing 10 to 20 per cent of the repolarisation amplitude. The approximate variation in position of the
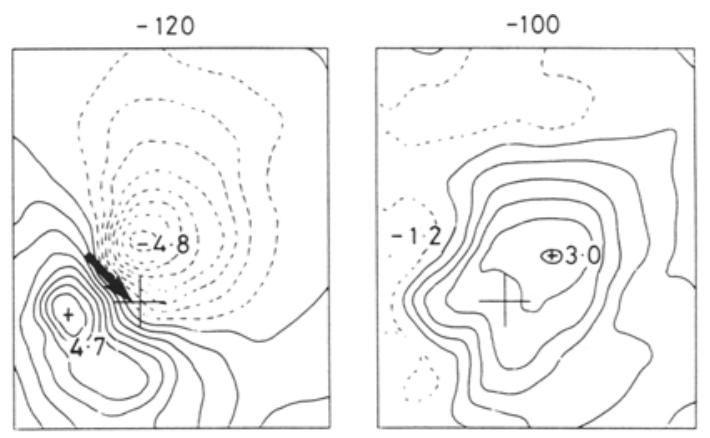

In Fig. 2 a sequence of eight spatial maps shows the

Examining the isofield contour maps of atrial repolarisa-

atrial repolarisation

Fig. 2 Magnetic isofield contour maps of atrial depolarisation and atrial repolarisation. Subject YT, 26 year-old-female. The top row shows four maps of atrial depolarisation patterns each $20 \mathrm{~ms}$ in time apart. The bottom row displays the atrial repolarisation patterns at four successive time instants before QRS-onset. Zero magnetic field is taken as the mean field during a $50 \mathrm{~ms}$ interval just before $P$-wave onset. The step size between successive contour lines is $600 f T$. The numbers inside the map frames give the extreme field values in $p T$ 
$-40$

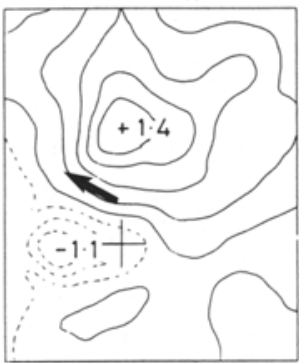

$-40$

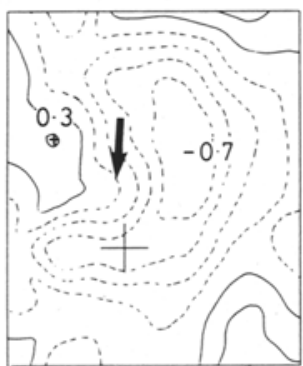

His-Purkinje activity

$-40$

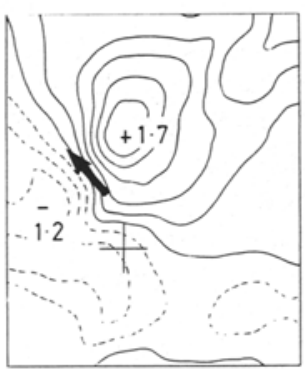

$-40$

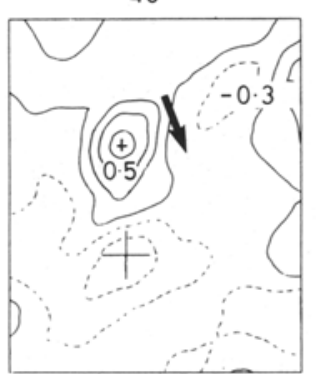

His-Purkinje activity
$-25$

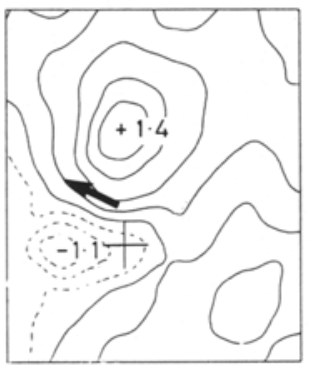

$-25$

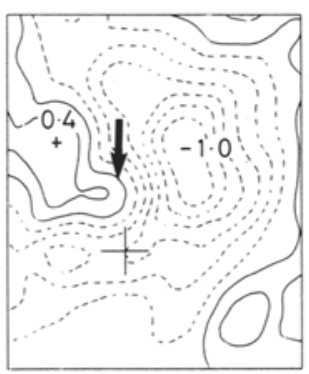

$-25$

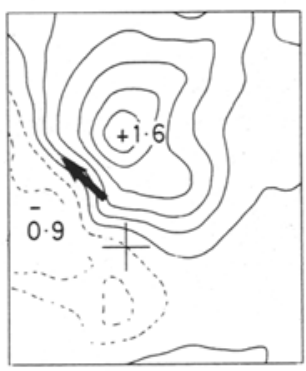

$-25$

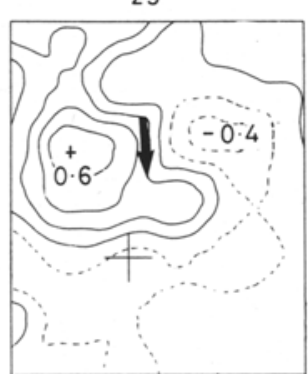

$-10$

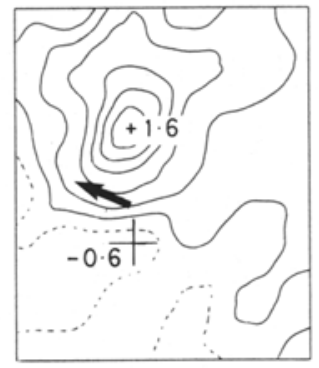

$-10$

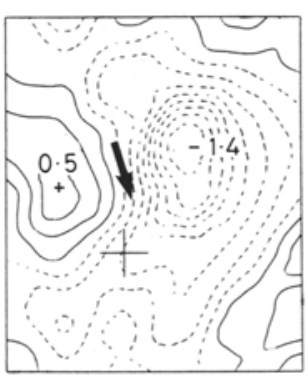

$-10$

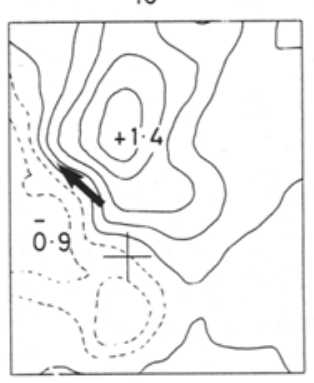

$-10$

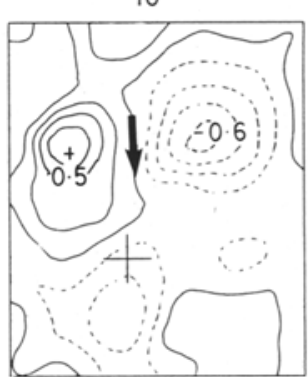

5

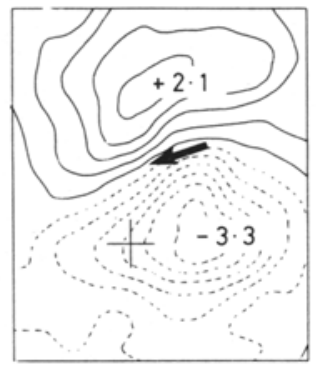

QRS - onset
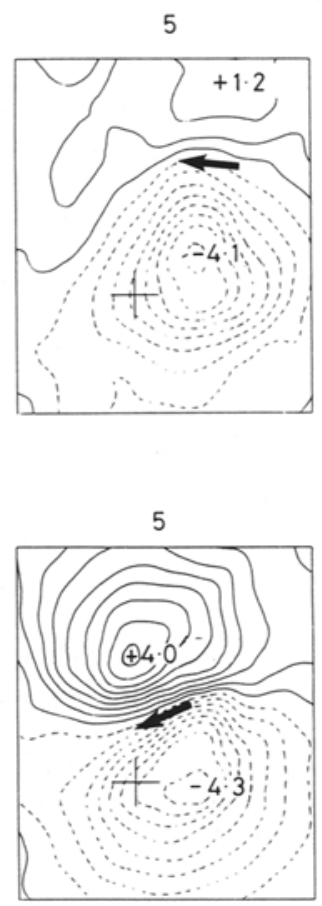

QRS - onset

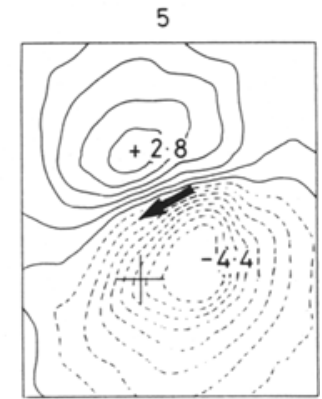

Fig. 3 Magnetic isofield contour maps of atrial repolarisation and His-Purkinje activation. (a) Subject BV, 65-year-old male. The top row shows three maps of atrial repolarisation patterns (contour step $300 \mathrm{fT}$ ) and one of early septal activation (contour step 500 fT), each map $15 \mathrm{~ms}$ later in time. The time segment during which the mean zero magnetic field was taken for these four maps is a 50 ms interval just before P-wave onset. The bottom row shows the resulting HPS-patterns (contour step 150f T) at the same time instants as the top row maps, after subtracting a typical mean atrial repolarisation map computed during a time segment from 60 until $50 \mathrm{~ms}$ before $Q R S$-onset. This typical repolarisation map is displayed as the most left map (contour step $300 f T$ ). The numbers inside the map frames give the extreme field values in $p T$. The numbers above the frames indicate the time instants in ms relative to $Q R S$-onset. (b) Subject $B V, 30$-year-old male. Contours the same as in (a)

contour indicating zero field during the PR-segment was in 15 of the 17 cases only about $10 \mathrm{~mm}$. To separate this slowly varying atrial repolarisation from underlying HPS activation a mean repolarisation map was computed over a time segment just after P-wave offset.

After atrial repolarisation subtraction dipolar magnetic fields patterns were found in 9 out of the 17 subjects. The amplitudes varied between $0 \cdot 1$ and $1.4 \mathrm{pT}$. The direction of the current dipole associated with these patterns was between downwards and down-leftwards, along the heart- axis and the location a few centimetres above and to the right compared with the initial septal activation dipole. For two subjects three maps of HPS-activity are displayed at time instants 40,25 and $10 \mathrm{~ms}$ prior to QRS onset (second row of Figs. $3 a$ and $3 b$, respectively). Also the initial septal activation pattern is given in the fourth map. For comparison the maps at the same time instants before the subtraction procedure showing merely atrial repolarisation activity are displayed in the first row. The most left map represents the mean atrial registration pattern over a 


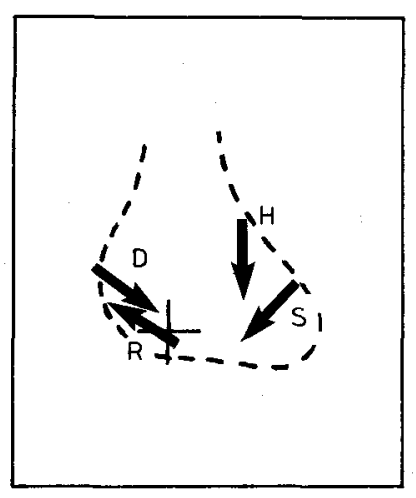

$10 \mathrm{~cm}$
B

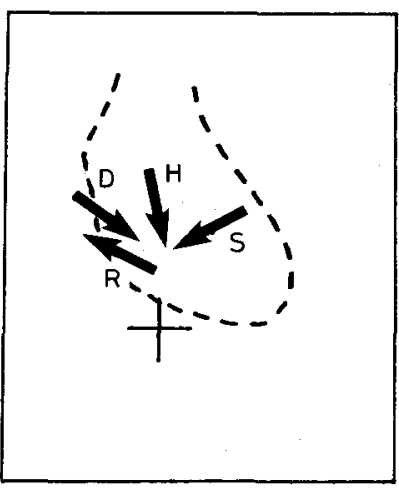

C

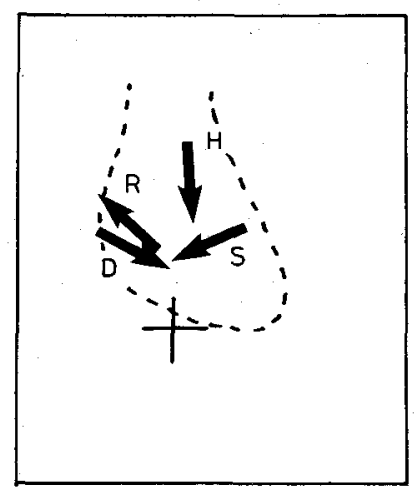

Fig. 4 Localisations of PR-interval phenomena. For the same three subjects as in Figs. 2 and 3 the locations and directions of the current dipoles associated with atrial depolarisation (D), atrial repolarisation ( $R$ ), His-Purkinje activation ( $H)$ and early septal activation (S) are displayed in one and the same frame. The third spatial parameter, the depth (measured from the pickup coil), is listed in Table 1. The dotted line indicates the heart silhouette as seen in an $X$-ray picture from each subject. Frames $A, B, C$ correspond to subjects $Y T(26 y), B V(65 y)$ and $B V(30 y)$, respectively

time segment $60-50 \mathrm{~ms}$ before QRS-onset, used in the subtraction procedure. In some cases not all of the atrial repolarisation activity could be removed, as can be seen from Fig. 3, where a negative field remains in the lower part of the grid.

The clear distinction between the four PR-interval phenomena, atrial depolarisation, atrial repolarisation, HPS activation and early septal activity, becomes evident when the position and the direction of the equivalent dipoles as found from the dipolar patterns are displayed in one and the same figure. This has been done in Fig. 4 for the same three subjects as in Figs. 2 and 3. The locations and directions of the four different electrical activities relative to each other are in good agreement with the anatomical reality, as can be seen by the displayed X-ray heart silhouette.

Table 1 Depths (in cm) of PR-interval phenomena

\begin{tabular}{lccc}
\hline & A & B & C \\
\hline Atrial depolarisation: D & $6 \cdot 3$ & $8 \cdot 5$ & $8 \cdot 0$ \\
Atrial repolarisation: R & $7 \cdot 2$ & $8 \cdot 0$ & $7 \cdot 2$ \\
His-Purkinje activation: H & $8 \cdot 0$ & $8 \cdot 3$ & $8 \cdot 0$ \\
Septal activation: S & $7 \cdot 7$ & $9 \cdot 6$ & $8 \cdot 8$ \\
\hline
\end{tabular}

\section{Discussion}

Before discussing the reported experimental results, we would like to accentuate a few aspects of our measuring method. The bandwidth reaches only $100 \mathrm{~Hz}$, whereas many others applied a high-frequency cutoff of $250 \mathrm{~Hz}$ (ERNÉ et al., 1985b; FENICI et al., 1985). But as BERBARI et al. (1979) reported, 80 per cent of the HPS-signal is below $50 \mathrm{~Hz}$. On the other hand, we did not use a high-pass filter to prevent the PR-segment from distortion due to the phase shift of the filter induced by the preceding P-wave or previous QRS-complex (BERBARI et al,, 1977). The low number of beats averaged (25) together with the low trigger jitter reduced the effect of averaging induced smoothening of the HPS signals. This fixed number of beats averaged for every measuring site implied a disadvantage, where we found a few of the 42 recordings of some subjects to have higher noise levels than others, due to temporarily increased magnetic environmental noise. This effect causes difficulties for the mapping procedure. A better method would therefore have been to use some criteria for the noise level of the online averaged complexes to stop averaging, rather than using a fixed number of beats to average at every grid site.
In most subjects we found dipolar patterns for maps taken during atrial depolarisation and maps taken during the PR-segment with roughly the same location for the current dipole situated above the atria but with opposite polarity. We conclude that the latter maps represent atrial repolarisation. With the experimentally found low variance in amplitude and even less variance in field pattern we think it is possible to eliminate a major part of this atrial repolarisation, by computing an average repolarisation map and subtracting it from maps during the last $40 \mathrm{~ms}$ of the PR segment. The segment that has to be chosen for computing this mean repolarisation map may neither contain atrial depolarisation nor HPS-activity, which however becomes difficult for subjects with a short PRsegment. In the case of nine of the 17 subjects dipolar field patterns were obtained, in which the location and direction of the current dipole strongly suggested that HPSactivation was the origin.

Finally, by determining the location and direction of the current dipoles of atrial depolarisation, atrial repolarisation, HPS-activation and early septal activation, we could clearly distinguish between all four of them. For most of the subjects the current dipole locations of these four PRinterval phenomena relative to each other were in reasonable agreement with functional anatomical knowledge of the heart.

The fact that the current dipoles of HPS activation differed in location and direction from the atrial repolarisation dipoles underlines the feasibility of the separation of both activities.

Acknowledgment-This study was supported by the Dutch Heart Foundation.

\section{References}

AкıмA, H. (1978) A method of bivariate interpolation and smooth surface fitting for irregularly distributed data points. ACM Trans. on Math. Software, 4, 148-159.

Berbari, E. J., Lazzara, R., Samet, P. and Scherlag, B. J. (1973) Noninvasive technique for detection of electrical activity during the PR segment. Circ., 48, 1005-1013.

Berbari, E. J., Scherlag, B. J. and Lazzarra, R. (1977) A computerized technique to record new components of the electrocardiogram. Proc. IEEE, 65, 799-802.

Berbari, E. J., Lazzara, R. and ScherlaG, B. J. (1979) The effects of filtering the His-Purkinje system electrocardiogram. IEEE Trans., BME-26, 82-85. 
ERnÉ, S. N., LehmanN, H. P., Masselli, M. and UchiKawa, Y. (1985a) Modelling of the His-Purkinje heart conduction system. In Biomagnetism: applications \& theory. WEINBERG, $\mathrm{H}$., Stroink, G. and Katila, T. (Eds.), Pergamon Press, New York, 126-131.

Erné, S. N., Fenici, R. R., Hahlbohm, H. D., Korsukewitz, J., LehmanN, H. P. and UChiKaWA, Y. (1985b). Magnetocardiographic study of the PR-segment of normals. In Biomagnetism: applications \& theory. WeInBERG, H., STronN, G. and Katila, T. (Eds.), Pergamon Press, New York, 132-136.

Farrell, D. E., TripP, J. H. and Norgren, R. (1978) Noninvasive information on the cardiac cycle: an assessment of the clinical potential of the electric and magnetic methods. Proc. $S P I E, 167,173-177$.

Farrell, D. E., TrIPP, J. H. and Norgren, R. (1980) Magnetic study of the His-Purkinje conduction system in man. IEEE Trans., BME-27, 345-349.

Fenici, R. R., Romani, G. L., Barbanera, S., Zeppilli, R., Carelli, P. and Modena, J. (1980) High resolution magnetocardiography: noninvasive investigation of His-Purkinje system activity in man. G. Ital. Cardiol., 10, 1366-1372.

Fenici, R. R., Masselli, M., Erné, S. N. and Hahlbohm, H. D. (1985) Magnetocardiographic mapping of the PR-interval phenomena in an unshielded hospital laboratory. In Biomagnetism: applications \& theory. WeInBERG, H., STroINK, G and Katila, T. (Eds.), Pergamon Press, New York, 137-141.

Horacek, B. M., Purcell, C., Lamothe, R., Merritt, R., Kafer, C., Periyalwar, S., Leon, L. J. and Stroink, G. (1986) The effect of torso geometry on magnetocardiographic isofield maps. Phys. Med. Biol., 32, 121-124.

Horan, L. G., Flowers, N. C. and SOHI, G. S. (1982) A dynamical electrical record of the pathway of human His-bundle activation from surface mapping. Circ. Res., 50, 47-54.

Lamothe, R., Stroink, G. and Gardner, M. J. (1987) High resolution magnetocardiographic studies of the PR-interval. 13th Conf. of CMBES, Halifax, Nova Scotia, Canada.

MacAulay, C. E., Stroink, G. and HoraCeK, B. M. (1985) Analysis of MCG spatial maps during the PR-interval. Med. \& Biol. Eng. \& Comput., 23, Suppl., Part 2, 1479-1480.

Peters, M. J., Swennenhuis, M. J. M., van Oosterom, A. and WeVERs-HenKe, J. J. (1982) The influence of inhomogeneities on the cardiac-magnetic-field distribution. Il Nuovo Cimento, 2D, 324-339.

Ros, H. H. (1976) Non invasive detection of His bundle activity. Ph.D. thesis, Free University of Amsterdam.

Scherlag, B. J., Lau, S. H., Helfant, R. H., Berkowitz, W. D., Stein, E. and Damato, A. N. (1969) Catheter technique for recording His bundle activity in man, Circ., 39, 13-18.

SNIJDER, W. V. (1978) Contour plotting. ACM Trans. on Math. Software, 4, 290-294.

\section{Authors' biographies}

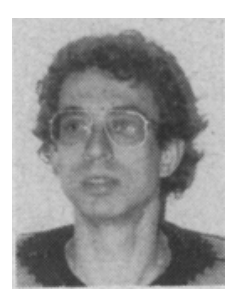

Ben J. ten Voorde was born on the 13th April 1956 in Hengelo, The Netherlands. He received his M.Sc. degree in Applied Physics in 1984 at the University of Technology, Twente. His thesis was about signal processing of biomagnetic recordings. From 1985 until summer 1986 he worked as a research assistant on high-resolution magnetocardiography in the same biomagnetism group of the Low Temperature Physics department. After this he joined the biomagnetism group of Dalhousie University in Canada to do a comparative study of body surface potential and magnetic mapping of non-Q myocardial infarctions.

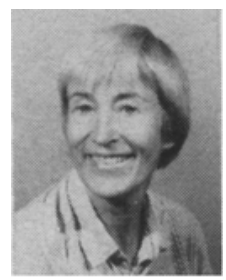

Maria Peters graduated in physics at the University of Leiden. She received her Ph.D. at Twente University. Her thesis was on magnetocardiography. Her current research interests include the experimental and theoretical development of biomagnetism, especially magnetoencephalography.

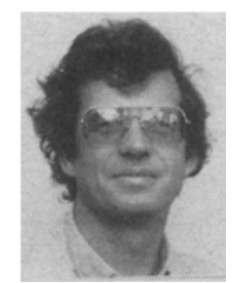

Gerhard Stroink was born on the 6th October 1942. He received his engineering degree in Physics from the Technical University of Delft, The Netherlands, and the Ph.D. degree in Physics from McGill University, Montreal, Canada. He joined the Department of Physics, Dalhousie University, Halifax, Canada, in 1973 and is presently an Associate Professor in that Department. He is also an adjunct Assistant Professor in the Physiology and Biophysics Department as well as an adjunct Associate Professor of the Technical University of Nova Scotia.

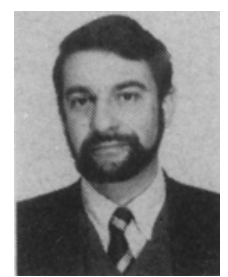

L. Ronald van der Wieken was born in London, UK, in June 1946. He received his medical education at the Municipal University of Amsterdam, The Netherlands and at Chaim Sheba Medical Center University of Tel Aviv, Israel. He received his cardiology training in the department of Professor Dr D. Durrer, University of Amsterdam. Presently he is practicing medicine in the Hospital Ziekenzorg Enschede, The Netherlands. 